\title{
Evolução temporal da dengue no município de Ribeirão Preto, São Paulo, 1994 a 2003
}

\author{
Temporal evolution of dengue fever in Ribeirão Preto, \\ São Paulo State, 1994 - 2003
}

\author{
Paula $\mathrm{Hino}^{1}$ \\ Carolina Carvalho dos Santos ${ }^{2}$ \\ M irian Oliveira dos Santos ${ }^{2}$ \\ Tarcísio N eves da Cunha ${ }^{4}$ \\ Claudia Benedita dos Santos ${ }^{2}$
}

${ }^{1}$ Universidade de São

Paulo. Av. Dr Enéas de

Carvalho Aguiar 419, Bela

Vista. 05403-000 São

Paulo SP.

paulahino@yahoo.com.br

${ }^{2}$ Escola deEnfermagem de

Ribeirão Preto,

UniversidadedeSão Paulo.

${ }^{3}$ UNESCO.
Abstract This epidemiological-descriptive study aimed to describe the temporal evolution of dengue cases in Ribeirão Preto, São Paulo State, between 1994 and 2003, according to month of occurrence and gender. Data were obtained from the compulsory notification forms provided by the Epidemiological Surveillance service of the Ribeirão Preto Municipal Health Secretary. We obtained incidence coefficients per 100,000 inhabitants, according to population estimates by the Brazilian Institute of Geography and Statistics. The city experienced a dengue epidemic in 2001, when the incidence coefficient reached 619.65 cases/100,000 inhabitants. We found 5553 cases during the study period, $0.07 \%$ of which occurred in 1994, 3.68\% in 1995, 4.52\% in 1996, $2.40 \%$ in $1997,1.82 \%$ in $1998,5.73 \%$ in 1999 , $3.75 \%$ in $2000,57.37 \%$ in $2001,6.25 \%$ in 2002 and $14.39 \%$ in 2003 . January to $M$ ay were the months with the highest occurrence levels. As to the gender variable, we found a proportion of approximately $1: 1$, showing small fluctuations between men and women for dengue cases during the entire study period. Results indicate the need to study the theme and reinforce the role of teaching institutions to tackle dengue in Brazil. Key words Denguefever, Epidemiology, Endemic diseases
Resumo Esteestudo caracteriza-seepidemiológico-descritivo com objetivo de descrever a evolução temporal dos casos de dengue em Ribeirão Preto, São Paulo, no período de 1994 a 2003, segundo mês deocorrência esexo. Os dados foram obti dos junto àsfichas de notificação compulsória fornecidas pela Vigilância Epidemiológica da Secretaria M unicipal de Saúde do município. Foram obtidos os coeficientes de incidência por 100.000 habitantes, segundo estimativas populacionais do Instituto Brasileiro de Geografia e Estatística. 0 município viveu uma epidemia de dengue no ano de 2001, quando o coeficiente deincidência chegou a 619,65 casos/ 100.000 habitantes, sendo quedentreos 5.553 casos encontradosno período estudado, 0,07\% ocorreram no ano de 1994, 3,68\% em 1995, 4,52\% em 1996, 2,40\% em 1997, 1,82\% em 1998, 5,73\% em 1999, 3,75\% em 2000, 57,37\% em 2001, 6,25\% em 2002 e, 14,39\% em 2003. Osmeses do ano demaior ocorrência da doença foram de janeiro a maio. Em relação à variável sexo, a proporção entre 0 número decasosfoi deaproximadamente 1:1, mostrando pequenas flutuações de casos de dengue entre homens e mulheres, para todo período estudado. Os resultadosapontam a necessi dade do desenvolvimento de estudos sobrea temática e reforçam o papel das instituições de ensino na questão da dengue no nosso país.

Palavras-chave Dengue, Epidemiologia, D oenças endêmicas 
Introdução

A dengueé uma doença febril aguda, cujo agente etiológico é um vírus do gênero Flavivírus. São conhecidos atualmente quatro sorotipos, antige nicamentedistintos: DEN 1, DEN 2, DEN 3 eDEN 4. Clinicamente, as manifestações variam de uma síndromeviral, inespecífica ebenigna, atéum quadro graveefatal dedoença hemorrágica com choque. Os fatores de risco para casos graves são: a cepa do sorotipo do vírus infectante, o estado imunitário e genético do paciente, a concomitância com outras doenças e a infecção prévia por outro sorotipo viral da doença ${ }^{1}$.

É de transmissão essencialmente urbana, ambiente no qual se encontram todos os fatores fundamentais para sua ocorrência: o homem, vírus, vetor eprincipalmenteas condiçõespolíticas, econômicas eculturais queformam a estrutura que permite o estabelecimento da cadeia de transmissão.

0 Aedes aegypti não é nativo das Américas; foi introduzido no Brasil a partir da África, provavelmente no inicio do século XIX. Tendo encontrado um meio ambiente adequado à sua sobrevivência e reprodução, ocupou o país no sentido de que progressivamente se expandiu geograficamente e aumentou a sua população. Foi erradicado do país em 1957, reintroduzido em 1967 e novamente eliminado em 1973. Finalmente, foi reintroduzido para iniciar a reocupação de seu antigo habitat em $1976{ }^{2}$. Acredita-se que o A edes aegypti tenha sido reintroduzido no país a partir de Salvador, Bahia, e estava presente em muitos países vizinhos. Países da América Central, M éxico, Venezuela, Colômbia, Suriname e alguns outros do Caribe já vinham apre sentando a doença desde os anos setenta ${ }^{1}$. Segundo a Organização M undial de Saúde(OM S), dois terços da população mundial estão sob risco de adquirir a doença e mais de cem países registram a presença dos dois tipos de mosquitos causadores e casos de doença ${ }^{3}$.

No Brasil, a primeira epidemia de dengue documentada clínica e laboratorialmente ocorreu em 1982, em Boa Vista, Roraima, com circulação dos sorotipos 1 e 4. A partir de 1986, foram registradas epidemias em vários estados, sendo a mais importantea ocorrida nos anos de 1986 e 1987 na cidade do Rio de Janeiro, onde pelos inquéritos sorológicos realizados, estimou-se pelo menos um milhão de pessoas infectadas pelo sorotipo 1. No ano de 2002 e 2003, foram notificados 794.219 e 341.776 casos, respectivamente 4 .

No Estado de São Paulo, o primeiro surto ocorreu em 1987 no município de Guararapes
( 30 casos notificados) e em Araçatuba ( 16 casos notificados ${ }^{5-6}$. Ribeirão Preto sofreu de novembro de 1990 a março de 1991 uma epidemia de dengue 1 com aproximadamente 8.900 casos confirmados, o que representou uma incidência de 546,9 casos/100.000 habitantes 7 . Desde então, a incidênciatem-semantido em índices baixos. De 1992 a 1994, houve um período de transmissão limitada, com poucos casos confirmados; em 1995, houve um recrudescimento da transmissão que continuou nos anos de 1996 e 1997, embora a níveis menos elevados do que a observada durante a epidemia.

Entre 1990 e 2000, os sorotipos 1 e2 disseminaram-se no país, com registro de epidemias principalmente nos grandes centros urbanos do Sudeste e N ordeste. Em 2003, os sorotipos 1, 2 e 3 foram isolados em 23 estados e observou-se um número crescente de internações por febre hemorrágica da dengue ${ }^{8}$. A série histórica de incidências da dengue no Brasil revela uma tendência ascendentede transmissão da doença, com valor máximo no ano de 2002. A ocorrência, até julho desse ano, foi de 385,1 casos/100 mil habitantes, com significativo aumento da forma hemorrágica da doença, com 2.090 casos. Hoje a doença está presente em 25 dos 27 estados brasileiros, e o mosquito vetor, em todos eles. 0 Estado de São Paulo seguiu a mesma tendência, sendo que a maior incidência ocorreu em 2001, com 136,2 casos/100.000 habitantes. As curvas de transmissão, tanto do Brasil como a do Estado de São Paulo, apresentaram comportamento cíclico, intercalando anos com incidências mais altas e anos com incidências mais baixas.

As razões para reemergência da dengue, atualmenteconsiderado um dos maiores problemas de saúde pública mundial, são complexas e não totalmente compreendidas. Como seu vetor, 0 Aedes aegypti, apresenta grande adaptação à vida urbana, sua propagação éprivilegiada devido aos hábitos consumistas modernos. A urbanização não planejada e o consequente aumento da densidade populacional, associado às condições precárias de saneamento básico, moradia inadequada e o livre trânsito e comércio entre países, proporcionaram condições favoráveis à transmissão do vírus ${ }^{9-10}$.

Nota-se que a organização atual do espaço dos grandes centros urbanos e a situação da população de mosquitos no país levou à conclusão de que a erradicação do Aedes aegypti de forma semelhante não é mais viável. 0 M inistério da Saúde (M S) passou então a recomendar o controlee não mais a erradicação. Controlesignifica 
a redução permanente da densidade vetorial, 0 que só será possível com a eliminação definitiva de criadouros que respondam por grande parte da reprodução do vetor ${ }^{2}$.

A presente investigação teve como objetivo descrever a evolução temporal dos casos de dengue em Ribeirão Preto (SP), no período de 1994 a 2003, segundo mês de ocorrência e sexo.

\section{Métodos}

Esteestudo caracteriza-se epidemiológico-descritivo. Foram utilizadas informações contidas nas fichas de notificação compulsória da dengue, as quais foram fornecidas pela Vigilância Epidemiológica (VE) da Secretaria Municipal de Saúde (SMS) do município de Ribeirão Preto(SP). Foram calculados os coeficientes de incidência por 100.000 habitantes para o período de 1994 a 2003. A população utilizada foi obtida no Instituto Brasileiro de Geografia e Estatística (IBGE). Foi elaborada uma planilha do Excel com os dados referentes aos casos de dengue, utilizando-se a técnica de dupla verificação.

Para realização do referido estudo, o projeto foi previamente submetido à apreciação e aprovação pelo Comitê de Ética em Pesquisa da Escola de Enfermagem de Ribei rão Preto da U niversidade de São Paulo. Por tratar-se de dados secundários, foi solicitado a dispensa do termo de consentimento livree esclarecido.
Resultados

A Tabela 1 mostra a distribuição dos casos de dengue no município, segundo mês de ocorrência, para cada um dos anos estudados. Pode-se observar que a maior ocorrência dos casos de dengue foi entre os meses dejaneiro a maio, sendo que os meses de março a maio foram os que registraram maior número de casos. 0 mês de junho do ano de 2001 apresentou um número elevado de casos (270); ressalta-se que 0 ano de 2001 foi 0 ano de maior incidência da doença no período estudado. Para os demais meses, o número de casos dedenguefoi relativamentebaixo.

A Tabela 2 apresenta a distribuição dos casos dedengueno município, segundo sexo, para cada um dos anos estudados. Para todos os anos estudados, a relação entre o número de casos, entre sexos, foi de aproximadamente 1:1, mostrando pequenas flutuações entre homens e mulheres. Houve duas perdas de informação para esta variável.

0 coeficiente de incidência dos casos de dengue em Ribeirão Preto foi de 8,7 casos/100.000 habitantes no ano de 1994, apresentando um aumento significativo nos dois anos seguintes para cercade 50 casos/ 100.000 habitantes. De 1997 a 1998, a incidência apresentou tendência de diminuição, voltando a se elevar no ano de 1999. Foi possível observar um pico da doença no ano de2001, período em queo coeficientedeincidência foi de 619,65 casos/ 100.000 habitantes, o que

Tabela 1. Distribuição dos casos de dengue segundo mês de ocorrência. Ribeirão Preto (SP). 1994 a 2003.

\begin{tabular}{lcrrrrrrrrrr}
\hline & \multicolumn{10}{c}{ Ano } \\
\cline { 2 - 10 } \multicolumn{1}{c}{ M ês } & 1994 & 1995 & 1996 & 1997 & 1998 & 1999 & 2000 & 2001 & 2002 & 2003 & Total \\
\hline Janeiro & 1 & 2 & 17 & 3 & 3 & 28 & 9 & 32 & 52 & 70 & 217 \\
Fevereiro & 0 & 11 & 15 & 26 & 4 & 62 & 21 & 79 & 82 & 86 & 386 \\
Março & 1 & 67 & 35 & 70 & 25 & 132 & 30 & 457 & 74 & 247 & 1138 \\
Abril & 2 & 75 & 100 & 28 & 32 & 75 & 72 & 1310 & 53 & 239 & 1986 \\
Maio & 0 & 36 & 68 & 6 & 33 & 19 & 48 & 961 & 56 & 112 & 1339 \\
Junho & 0 & 2 & 10 & 0 & 1 & 1 & 19 & 270 & 7 & 30 & 340 \\
Julho & 0 & 5 & 2 & 0 & 1 & 1 & 1 & 60 & 0 & 10 & 80 \\
Agosto & 0 & 2 & 0 & 0 & 0 & 0 & 6 & 8 & 0 & 2 & 18 \\
Setembro & 0 & 0 & 0 & 0 & 0 & 0 & 0 & 5 & 0 & 1 & 6 \\
Outubro & 0 & 0 & 0 & 0 & 0 & 0 & 0 & 3 & 0 & 0 & 3 \\
Novembro & 0 & 2 & 1 & 0 & 0 & 0 & 1 & 1 & 7 & 0 & 12 \\
Dezembro & 0 & 3 & 3 & 0 & 2 & 1 & 1 & 0 & 16 & 2 & 28 \\
Total & 4 & 205 & 251 & 133 & 101 & 319 & 208 & 3186 & 347 & 799 & 5553 \\
\hline
\end{tabular}

Fonte: Vigilância Epidemiológica, Secretaria M unicipal da Saúde, Ribeirão Preto. 
faz lembrar a epidemia ocorrida no município nos anos de 1990 e 1991. Em 2002, houve um declínio significativo, entretanto, o coeficientede incidência volta a se elevar em 2003, conforme ilustrado no Gráfico 1.

\section{Discussão}

0 primeiro questionamento a ser feito é se há possibilidade de atingir o objetivo proposto ao se utilizar informações produzidas pelo sistema de vigilância, visto que as informações baseadas

Tabela 2. Distribuição dos casos de dengue segundo sexo. Ribeirão Preto (SP). 1994 a 2003.

\begin{tabular}{rrrcrr}
\hline & \multicolumn{4}{c}{ Sexo } & \\
\cline { 2 - 4 } Ano & masculino & feminino & não informado & Total \\
\hline 1994 & 3 & 1 & 0 & 4 \\
1995 & 85 & 119 & 1 & 204 \\
1996 & 121 & 130 & 0 & 251 \\
1997 & 76 & 57 & 0 & 133 \\
1998 & 46 & 54 & 1 & 100 \\
1999 & 140 & 179 & 0 & 319 \\
2000 & 85 & 123 & 0 & 208 \\
2001 & 1427 & 1759 & 0 & 3186 \\
2002 & 169 & 178 & 0 & 347 \\
2003 & 377 & 422 & 0 & 799 \\
Total & 2529 & 3022 & 2 & 5551 \\
\hline
\end{tabular}

Fonte: Vigilância Epidemiológica, Secretaria M unicipal da Saúde, Ribeirão Preto.

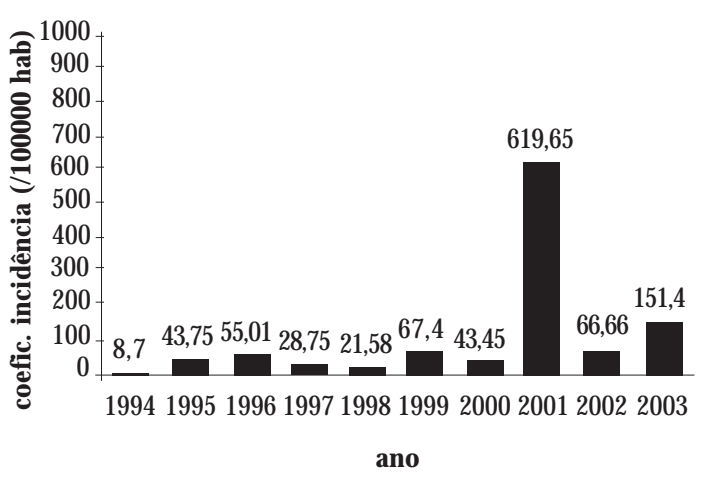

Gráfico 1. Coeficiente de incidência (por 100.000 habitantes) da dengue. Ribeirão Preto (SP), 1994 a 2003.

Fonte: Vigilância Epidemiológica, Secretaria M unicipal da Saúde, Ribeirão Preto. em casos notificados mostram apenas uma parte da realidade, pois ésabido que muitas pessoas infectadas ou assintomáticas ou mesmo queapresentem sintomas não chegam a fazer parte das estatísticas oficiais ${ }^{11}$. Portanto, há a necessidade de se gerar dados com qualidade e agilidade para quedemandem ações efetivas de prevenção e controle da dengue.

Dentro da perspectiva de queas ações de controle da dengue baseiam-se nas informações disponibilizadas pelo sistema de vigilância, única ferramenta disponível no momento em que os casos ocorrem, a presente análise buscou um conhecimento da situação da dengue em Ribeirão Preto, fornecendo, assim, informações para otimizar o controle da doença no município.

0 estudo da dengue no município de Ribeirão Preto no período de 1994 a 2003 retratou diversos períodos da doença, sendo que, no ano de 1995, apresentou um aumento significativo em relação ao ano anterior, diminuindo apenas em 1997 e evidenciando um novo aumento dos casos no ano de 1999.

No ano de 1997, foi realizado estudo em uma instituição correcional de adolescentes localizada naregião periférica deRibeirão Preto, ondefoi constatado um surto de dengue que se iniciou entre internosefuncionários dessa instituição. Foi realizado inquérito sorológico e virológico da população de internos e funcionários, sendo que a incidência foi de $23,8 \%$ e $18,6 \%$, respectivamente ${ }^{12}$.

A explosão dos casos de dengue ocorrida no ano de2001 com coeficientedeincidência de 619,65 casos/ 100.000 habitantes veio seguida de uma redução significativa no ano seguinte, quando foi verificado um coeficiente de incidência de 66,66 casos/100.000 habitantes. Tal fato pode ser decorrente deum possível esgotamento de susceptíveis eredução sazonal da densidade vetorial, conforme ocorrido no município do Rio de Janeiro ${ }^{2}$ e/ou resultado das ações dos agentes de vetores.

Os resultados do estudo evidenciaram a ocorrência de um maior número de casos nos meses de janeiro a maio (91,22\%); ressalta-se que este período é marcado por chuvas, altas temperaturas, altitudes eventos, dados estes bastante discutidos quando se trata da transmissão da dengue.

No Estado de São Paulo, verificou-se que a temperatura atuou como fator modelador do processo de infestação por A edes aegypti de várias regiões. Acredita-se que a temperatura influencia na transmissão da dengue, pois interfere nas atividades de repasto sanguíneo das fêmeas dos mosquitos, em sua longevidade e no período de incubação do vírus. Embora as chuvas es- 
tejam associadas com a incidência de dengue, há controvérsias sobre o assunto. Sendo o Aedes aegypti um vetor marcadamente domiciliado, utiliza diversos criadouros cuja água independe da chuva e, dessa forma, são menos afetados pela sazonalidade ${ }^{13}$.

Estudo realizado no município de São José do Rio Preto com agentes de controle de vetores evidenciou que poderia ser atribuído aos meses de janeiro efevereiro grande parte das dificuldades relacionadas ao trabalho dos agentes, sendo as debilidades as seguintes: ocorrência de chuvas, pouca adesão às práticas pelos moradores, aumento do número de criadouros e larvas, período de férias e festas e aumento do número de casas fechadas e a presença constante do serviço nas casas $^{14}$.

Vários são os desafios colocados ao controle desta endemia. Uma primeiraquestão équeainda predominam no Brasil os modelos médico-assistencialista e assistencial sanitarista em saúde pública e a promoção da saú de que, segundo a Carta de Ottawa, é"o processo de capacitação da comunidade para atuar na melhoria da qualidade de vida e saúde, incluindo uma maior participação no controle desse processo", só ocorrerá a partir de alterações no sistema de saúde vigente ${ }^{14}$. N este modelo, pouco se tem privilegiado a prevenção, entendida como a atuação sobre os riscos deacon- tecer um evento não desejado e a antecipação das ações focadas na saúde e não na doença.

A prevenção da doença representa um desafio para os serviços de saúde, pois atualmente se resume no combate ao vetor, o Aedes aegypti, único transmissor com importância epidemiológica nas Américas. Para diminuir os criadouros do vetor, o M S preconiza o saneamento ambiental e o controle químico. A questão da dengue é de grande complexidade; portanto, surge a necessidade de repensar a atual estratégia de controleda doença, deven do envolver outros setores da sociedade, particularmente na questão de reforçar a educação em saúde, visando informar à população sobre a doença, o vetor, as medidas preventivas e na melhoria das condições de urbanização e habitação, coleta regular de lixo e abastecimento de água encanada.

Os resultados obtidos apontam a necessidade de investir em pesquisas que esclareçam questões relativas a diversos fatores envolvidos na transmissão da dengue, sua distribuição espacial, desenvolvimento de vacinas, entre outros. Deve-se ressaltar a importância das instituições de ensino, pois estas têm grande contribuição a oferecer na formação dejovens, com vistas à promoção da saúde, tornando-os cidadãos conscientes sobre sua responsabilidade com o meio e sociedade a que estão inseridos.

\section{Colaboradores}

P Hino foi responsável pela análise/discussão dos dados e redação do artigo. M O Santos e CC Santos trabal haram na construção do projeto e coleta dos dados. CB Santos definiu a metodologia do estudo, colaborou no processamento e análise dos dados, bem como na revisão final do artigo. TN Cunha colaborou na revisão final do texto.

\section{Agradecimentos}

À Fundação de Amparo à Pesquisa do Estado de São Paulo (FAPESP); ao Conselho Nacional de Desenvolvimento Científico e Tecnológico (CN Pq); eà Vigilância Epidemiológica da Secretaria M unicipal deSaúdedo município de Ribeirão Preto (SP). 


\section{Referências}

1. Tauil PL. Urbanização e ecologia do dengue. Cad Saude Publica 1001; 17(Sup 1):99-102.

2. Penna MLF. Um desafio para a saúde pública brasileira: o controle do dengue. Cad Saude Publica 2003; 19(1):305-309.

3. Word Health Organization. Report on global surveillance of epidemic prone infectious diseases. Dengue and dengue hemorrhagic fever. [acessado 2006 jul 20]. Disponível em http://ww.who.int/emcdocuments/surveillance/docs/whocdscsrisr.html/ dengue.dengue.htm

4. Domingos M F. Aspectos da ecologia de Aedes aegypti (Linnaeus) em Santos, São Paulo, Brasil [tese]. São Paulo (SP): Faculdade de Saúde Pública; 2005.

5. Pontes RJS, Ruffino-N etto A. Dengue em localidade urbana da região sudeste do Brasil: aspectos epidemiológicos. Rev. Saude Publica 1994; 28(3):218-227.

6. Lefèvre $F$, Lefèvre AM, Scabdar SAS, Yassumaro $S$ Representações sociais sobre relações entre casos de plantas e 0 vetor da dengue. Rev. Saude Publica 2004; 38(3):405-414.

7. Pontes RSJ. Estudo da epidemia de dengue no município de Ribeirão Preto-SP, 1990-1991 [tese]. Ribeirão Preto (SP): Faculdade de M edicina de Ribeirão Preto, Universidade de São Paulo; 1992.

8. Duarte HHP, França EB. Qualidade dos dados da vigilância epidemiológica da dengue em Belo Horizonte, M G. Rev. Saude Publica 2006; 40(1):134-142.

9. Costa AIP, Natal D. Distribuição espacial da dengue e determinantes socioeconômicos em localidade urbana no Sudeste do Brasil. Rev. Saude Publica 1998; 32(3):232-236.
10. Toledo ALA, Escosteguy CC, Medronho RA, Andrade FC. Confiabilidade do diagnóstico final de dengue na epidemia 2001-2002 no M unicípio do Rio de Janeiro, Brasil. Cad Saude Publica 2006; 22(5):933-940.

11. Mondini A, Chiaravalloti-N eto F, Sanches M G, Lopes JCC. Análise espacial da transmissão de dengue em cidade de porte médio do interior paulista. Rev. Saude Publica 2005; 39(3):444-451.

12. Rodrigues EMS, Dal-Fabbro AL, Salomão R, Ferreira IB, Rocco $M$, Fonseca BAL. Epidemiologia da infecção pela dengue em Ribeirão Preto, SP, Brasil. Rev. Saude Publica 2002; 36(2):160-165.

13. Donalísio M R, Glasser CM. Vigilância Entomológica e Controle de Vetores do Dengue. Rev. bras. epidemiol. 2002; 5(3):259-272.

14. Baglini V, Favaro EA, Ferreira AC, ChiaravallotiNeto F, Mondini A, Dibo M, Barbosa AAC, Ferraz $A A$, Cesarino MB. Atividades de controle do dengue na visão de seus agentes e da população atendida, São José do Rio Preto, São Paulo, Brasil. Cad Saude Publica 2005; 21(4):1142-1152.

Artigo apresentado em 17/12/2006

Versão final apresentada em 28/06/2007 